\title{
The Effects of Removable Denture on Swallowing
}

\author{
Dae-Sik Son, $\mathrm{MD}^{1}$, Jin Wan Seong, $\mathrm{MD}^{1}$, Younghoon Kim, $\mathrm{BS}^{2}$, \\ Youngjoon Chee, $\mathrm{PhD}^{2}$, Chang Ho Hwang, $\mathrm{MD}^{1}$
}

\author{
${ }^{1}$ Department of Physical Medicine and Rehabilitation, Ulsan University Hospital, University of \\ Ulsan College of Medicine, Ulsan; ${ }^{2}$ Department of Electrical Engineering, University of Ulsan, Ulsan, Korea
}

\begin{abstract}
Objective To investigate the relationship between removable dentures and swallowing and describe risks.
Methods Twenty-four patients with removable dentures who were referred for videofluoroscopic swallowing study (VFSS) were enrolled. We evaluated the change of swallowing function using VFSS before and after the removal of the removable denture. The masticatory performance by Kazunori's method, sensation of oral cavity by Christian's method, underlying disease, and National Institutes of Health Stroke Scale for level of consciousness were collected. Functional dysphagia scales, including the oral transit time (OTT), pharyngeal transit time (PTT), percentage of oral residue, percentage of pharyngeal residue, oropharyngeal swallow efficiency (OPSE), and presence of aspiration were measured.

Results Four patients dropped out and 20 patients were analyzed (stroke, 13 patients; pneumonia, 3 patients; and others, 4 patients). The mean age was $73.3 \pm 11.4$ years. There were significant differences before and after the removal of the denture for the OTT. OTT was significantly less after the removal of the denture (8.87 vs. 4.38 seconds, $\mathrm{p}=0.01)$. OPSE increased remarkably after the removal of the denture, but without significance (18.24\%/ sec vs. $25.26 \% / \mathrm{sec}, \mathrm{p}=0.05$ ). The OTT and OPSE, while donning a removable denture, were correlated with the masticatory performance (OTT, $p=0.04$; OPSE, $p=0.003$ ) and sensation of oral cavity (OTT, $p=0.006$; OPSE, $p=0.007$ ). Conclusion A removable denture may have negative effects on swallowing, especially OTT and OPSE. These affects may be caused by impaired sensation of the oral cavity or masticatory performance induced by the removable denture.
\end{abstract}

Keywords Dysphagia, Removable partial denture, Swallowing

Received June 22, 2012; Accepted September 19, 2012

Corresponding author: Chang Ho Hwang

Department of Physical Medicine and Rehabilitation, Ulsan University Hospital, University of Ulsan College of Medicine, 877 Bangeojinsunhwan-doro, Dong-gu, Ulsan 682-714, Korea

Tel: +82-52-250-7210, Fax: +82-52-250-7211, E-mail: chhwang1220ciba@ yahoo.co.kr

(c) This is an open-access article distributed under the terms of the Creative Commons Attribution Non-Commercial License (http://creativecommons. org/licenses/by-nc/3.0) which permits unrestricted noncommercial use, distribution, and reproduction in any medium, provided the original work is properly cited.

Copyright $\odot 2013$ by Korean Academy of Rehabilitation Medicine

\section{INTRODUCTION}

Elderly patients often complain of declines in masticatory functions due to loss of teeth. Such patients frequently wear dentures for mechanical gains, but improper denture wear causing sensory declines occur on and around teeth can result in increased swallowing duration [1]. Sensory inputs in the oral cavity including taste are important in voluntary swallowing, and changes in tastes or sensory declines in the oral cavity cause remarkable changes in swallowing behavior [2]. Sensory changes occur after strokes [3], and these sensory changes raise the 
risk of aspiration [4]. Changes in oral cavity environments and diseases that can cause oral sensory declines can cause dysphagia. We planned the present study to examine the association between denture wearing and dysphagia and identify risk factors for dysphagia in patients who use removable dentures.

\section{MATERIALS AND METHODS}

\section{Subjects}

The present study is a prospective, single group, clinical series study conducted with patients using removable dentures among those patients for whom videofluoroscopic swallowing study (VFSS) was requested at the department of physical medicine and rehabilitation of Ulsan University Hospital between March 2011 and February 2012. Patients who were using non-removable dentures (including implants), those who had any history of jaw arthritis, past history of anatomical damage, defects, or history of surgery on the laryngopharyngeal region and those who did not agree to participate in the study were excluded. The present study passed a deliberation by the Institutional Review Board for clinical studies (UUH 11-23) and was conducted after receiving written agreements from the patients or their protectors.

\section{Methods}

Histories was taken for all the subjects regarding underlying diseases, and the subjects' state of consciousness were classed into $0,1,2$, and 3 in accordance with the National Institutes of Health Stroke Scale (NIHSS)-1a.

Evaluation of masticatory performance and sensation of oral cavity

To evaluate masticatory performance, the study method proposed by Kazunori et al. [5] was used with some modifications. After asking the subjects to chew a $2.5 \mathrm{~cm} \times 1.5$ $\mathrm{cm} \times 1.0 \mathrm{~cm}$ sized commercialized soft candy 30 times and spit it out, the gathered candy fragments were rinsed with flowing water to remove saliva. The candy fragments were put into $20 \mathrm{~mL}$ of distilled water and stirred for two minutes with a tongue depressor. The supernatant liquid was collected and the concentration of dissolved glucose was measured with a blood sugar meter. To eliminate the effects of temperature and humidity, the concentrations before and after mastication were compared at the time of every test to measure increment in the surface area of masticated candies. The subjects were divided into three groups: a group in which the surface area increased by $0 \%-25 \%$, a group in which the surface area increased by $25 \%-50 \%$, and a group in which the surface area increased by $50 \%$ or more.

To evaluate sensation of oral cavity, the two point discrimination test proposed by Guilleminault et al. [6] was used with modifications. The two point discrimination test is a composite sensory test used to measure when the distance before two points is felt as one point when two points are stimulated simultaneously. The method is to measure a distance at which the subjects could easily distinguish two points first and then gradually decrease the distance. Needles with non-sharp ends were fixed to pincers and the distances at the left/right side walls of the palate and the basal region of the uvula in random order were measured. The distances were measured in a unit of $1 \mathrm{~mm}$ and the measurement was conducted two times at each site, six times in total. There were interval distance of at least $2 \mathrm{~mm}$ at each session and the subject took a rest for two minutes after each session. The averages were obtained and the subjects were divided into three groups: a group of shorter than $10 \mathrm{~mm}$, a group from 10 to shorter than $15 \mathrm{~mm}$, and a group of longer than $15 \mathrm{~mm}$.

History taking, NIHSS and evaluation of masticatory performance and sensation of oral cavity were conducted by one researcher before the VFSS was conducted.

\section{Quantitative analysis using VFSS}

VFSSs were conducted on the same day at the same place [7]. The aforementioned studies have high inter and intra test reliability. To avoid errors in the results of the VFSS due to nasogastric tubes, nasogastric tubes were removed from all the patients 20 minutes before the studies, and after removing secretions in the oral cavity and decompressing balloons in tracheostomy patients. One specialist in dysphagia in the rehabilitation medicine department implemented modified Logeman's protocol [8]. The protocol was implemented after conducting the fluoroscopy when the patient was sitting laterally at the fluoroscope so that accurate anatomical structures were shown and while video-recording the fluoroscopic screens. In each study, dysphagia diet I, a mixture of $80 \mathrm{~g}$ of yogurt and $10 \mathrm{~g}$ of water-soluble barium, was used and the study began with the doctor's instruction, "Swallow 
it" after putting $30 \mathrm{~g}$ of the diet at a time into the mouth of the patient using a spoon. The study was conducted one time when the patient was wearing dentures. The dentures were removed, any test medium remaining in the pharyngolarynx was removed using coughing and double swallowing, any test medium remaining in the oral cavity was spit out and the oral cavity was rinsed using normal saline. The same process was repeated after a resting time of five minutes. After the study, the video was played, motions were captured and 2D quantitative analyses were conducted using Adobe Photoshop CS3 (Adobe Systems Inc., San Jose, CA, USA) by the Department of Electrical Engineering, University of Ulsan. The produced data were read by a physiatrist. To avoid decreases in measured values due to masking caused by the radiopacity of dentures, the maximum area of the test diet gathered on the tongue when the mouth was open before swallowing began was measured and the maximum area of the test diet in the oral cavity when the mouth was open after swallowing was completed was measured. The definitions of the measured variables are as follows. Oral cavity transit time (OTT) is the time (second) from the moment at which the major bolus in the oral cavity began to move posterior to the moment at which the front end of the bolus arrived at the point of crossing between the lower edge of mandibular ramus and the tongue base, pharyngeal transit time (PTT) is the time (second) from the moment at which the front end of the bolus arrived at the point of crossing between the lower edge of mandibular ramus and the tongue base to the moment at which the rear end of the bolus passed the cricopharyngeus, oral cavity residue is the percentage of bolus remaining in the oral cavity, pharyngeal residue is the percentage of bolus remaining in the pharynx, oropharyngeal swallow efficiency (OPSE) is the value obtained by dividing the fraction of bolus swallowed through the esophagus by the total time taken to pass the oropharynx and aspiration is the passing through the vocal fold of foods [9].

\section{Statistics}

Data were statistically analyzed using SPSS ver. 17.0 (SPSS Inc., Chicago, IL, USA). The significance level ( $\alpha$ ) was set to 0.5 based on p-value $<0.05$. The power was specified at $80 \%$ and the drop-out rate was predicted at $20 \%$. The OPSE index data in this author's previous study [9] were used to calculate the standardized difference of
2 using the minimum value of mean differences at $2 \%$ / sec and the standard deviation of reaction differences at $2 \% / \mathrm{sec}$. The sample size requirement of 20 was obtained using the Altman's nomogram and the drop rate was compensated to calculate the number of patients necessary for the study at 24 . Values obtained by log substituting individual measured values were used. Normality was checked using the normal quantile-quantile plot, histograms, and the Kolmogorov-Smirnov test. Nonparametric methods were used in for non-normally distributed data. Changes in the OTT, PTT, percentage of oral residue, percentage of pharyngeal residue and OPSE were analyzed using paired Student's t-tests in relation to whether dentures were worn or not. For variables showing significant changes among the aforementioned variables were analyzed among subgroups of the state of consciousness, underlying disease, masticatory performance and sensation of oral cavity using the non-parametric Kruskal-Wallis.

\section{RESULTS}

\section{Demographic information on the study subjects}

The mean age of the study subjects was $73.3 \pm 11.42$ years and the study subjects consisted of 10 males and 10 females. As underlying diseases, 13 had stroke, 3 had pneumonia, and 4 had other diseases. As for the state of consciousness, under NIHSS-1a, 15 subjects score 0,4 scored 1 , and 1 subject 2 . The masticatory performance of the subjects were analyzed, and 7 had less than $25 \%$ abilities, 9 had $25 \%-50 \%$ abilities and 4 had $50 \%$ or higher abilities. Sensation of oral cavity were analyzed and 8 subjects showed shorter than $10 \mathrm{~mm}$ distances, 4 subjects showed $10-15 \mathrm{~mm}$ and 8 showed $15 \mathrm{~mm}$ or longer distances (Table 1).

Changes in functional swallowing/dysphagia scales in relation to whether dentures were worn or not

Changes in functional dysphagia scales were examined in individual patients and according to the results, OTTs were shortened when dentures were removed compared to when dentures were worn and the differences were statistically significant ( 8.87 vs. 4.38 seconds, $\mathrm{p}=0.013$ ). OPSE tended to increase when dentures were removed compared to when dentures were worn although the differences were not statistically significant $(18.24 \% / \mathrm{sec}$ 
vs. $25.26 \% / \mathrm{sec}, \mathrm{p}=0.053)$. PTTs, OTTs and percentage of pharyngeal residues did not differ significantly between when dentures were removed and when dentures were worn (Table 2).

Changes in aspiration between when dentures were worn and when dentures were removed

When dentures were worn, four out of the entire $20 \mathrm{sub}$ jects showed aspiration (20\%). Although no statistically significant differences were seen between the groups, when dentures were removed, aspiration occurred only in two out of the four who showed aspiration when den-

Table 1. Demographic characteristics of subjects $(n=20)$

\begin{tabular}{lc}
\hline \multicolumn{1}{c}{ Characteristic } & No. $(\%)$ \\
\hline Age (yr), mean \pm SD (range) & $73.3 \pm 11.42(52-90)$ \\
\hline Sex (male:female) & $10: 10$ \\
Preceding disease & $13(65)$ \\
\hline Stroke & $3(15)$ \\
\hline Pneumonia & $4(20)$ \\
\hline Others & \\
\hline Level of consciousness (NIHSS-1a) & $15(75)$ \\
\hline 0 & $4(20)$ \\
\hline 1 & $1(5)$ \\
\hline 2 & $7(35)$ \\
\hline Masticatory performance (\%) & $9(45)$ \\
\hline$<1.25$ & $4(20)$ \\
\hline $1.25-1.5$ & $8(40)$ \\
\hline$\geq 1.5$ & $4(20)$ \\
\hline Sensation of oral cavity (mm) & $8(40)$ \\
\hline$<10$ & \\
\hline $10-15$ & \\
\hline
\end{tabular}

SD, standard deviation; NIHSS, National Institutes of Health Stroke Scale. tures were worn $(10 \%)$.

Relationships between OTT/OPSE and the state of consciousness/masticatory performance/sensation of oral cavity/underlying disease

OTTs that were statistically significantly shortened when dentures were removed compared to when dentures were worn and OPSE that tended to increase although significantly were analyzed separately for subgroups divided on the state of consciousness, masticatory performance, sensation of oral cavity and underlying disease. The OTTs and OPSE measured when dentures were worn showed significant differences among the levels of the patients' masticatory performance (OTT, $\mathrm{p}=0.04$; OPSE, $\mathrm{p}=0.003$ ). The OTTs and OPSE measured when dentures were worn also showed significant differences among the levels of the patients' sensation of oral cavity (OTT, $p=0.006$; OPSE, $p=0.007$ ). The OTTs and OPSE measured when dentures were worn did not show any significant associations with the states of consciousness or underlying disease (Table 3).

\section{DISCUSSION}

The present study attempted to examine the effects of removable denture on swallowing and utilize the results in rehabilitation of dysphagia patients. Changes in functional dysphagia scales between when dentures were worn and when dentures were removed were observed and OTTs were statistically significantly shortened when dentures were removed compared to when dentures were worn. OPSE showed tended to increase although not statistically significant. The OTTs and OPSE differed significantly among the levels of masticatory performance and the threshold values of sensation of oral cavity. Based on these results, the delays in OTTs and the decreases in

Table 2. Comparison of the functional dysphasia scale

\begin{tabular}{lccccc}
\hline & $\operatorname{logOTT}$ & $\operatorname{logOPSE}$ & $\operatorname{logPTT}$ & $\operatorname{logORES}$ & $\operatorname{logPRES}$ \\
\hline WD & $0.73 \pm 0.44$ & $1.09 \pm 0.40$ & $0.15 \pm 0.52$ & $1.06 \pm 0.48$ & $1.22 \pm 0.46$ \\
WOD & $0.37 \pm 0.49$ & $1.26 \pm 0.38$ & $0.26 \pm 0.55$ & $1.13 \pm 0.37$ & $1.25 \pm 0.43$ \\
WD-WOD & $0.35 \pm 0.57$ & $-0.17 \pm 0.37$ & $-0.11 \pm 0.56$ & $-0.06 \pm 0.40$ & $-0.03 \pm 0.37$ \\
p-value & 0.013 & 0.053 & 0.393 & 0.607 & 0.757 \\
\hline
\end{tabular}

Values are mean \pm standard deviation.

OTT, oral transit time; OPSE, oropharyngeal swallow efficiency; PTT, pharyngeal transit time; ORES, percentage of oral residue; PRES, percentage of pharyngeal residue; WD, with denture; WOD, without denture. 
Table 3. Relationship between functional dysphasia scale and level of consciousness, masticatory performance, sensation of oral cavity, and underlying disease

\begin{tabular}{|c|c|c|c|c|}
\hline & $\operatorname{logOTT}$ WD & logOTT WOD & logOPSE WD & logOPSE WOD \\
\hline \multicolumn{5}{|l|}{ NIHSS-1a } \\
\hline 0 & 9.87 & 11.27 & 11.60 & 10.80 \\
\hline 1 & 11.25 & 5.25 & 7.75 & 11.75 \\
\hline 2 & 17.00 & 20.00 & 5.00 & 1.00 \\
\hline $\mathrm{p}$-value & 0.486 & 0.050 & 0.325 & 0.247 \\
\hline \multicolumn{5}{|c|}{ Underlying disease } \\
\hline Pneumonia & 11.33 & 13.33 & 6.00 & 5.33 \\
\hline Stroke & 10.62 & 8.54 & 10.92 & 11.92 \\
\hline Others & 9.50 & 14.75 & 12.50 & 9.75 \\
\hline p-value & 0.915 & 0.124 & 0.323 & 0.212 \\
\hline \multicolumn{5}{|c|}{ Masticatory performance (\%) } \\
\hline$<1.25$ & 14.71 & 10.71 & 4.57 & 7.29 \\
\hline $1.25-1.5$ & 7.22 & 9.89 & 14.78 & 13.56 \\
\hline$\geq 1.5$ & 10.50 & 11.50 & 11.25 & 9.25 \\
\hline $\mathrm{p}$-value & 0.043 & 0.896 & 0.003 & 0.098 \\
\hline \multicolumn{5}{|c|}{ Sensation of oral cavity (mm) } \\
\hline$<10$ & 8.25 & 11.88 & 13.88 & 11.25 \\
\hline $10-15$ & 5.00 & 4.75 & 14.00 & 14.75 \\
\hline$\geq 15$ & 15.50 & 12.00 & 5.38 & 7.63 \\
\hline $\mathrm{p}$-value & 0.006 & 0.094 & 0.007 & 0.130 \\
\hline
\end{tabular}

Values are average of the ranks.

NIHSS, National Institutes of Health Stroke Scale; OTT, oral transit time; OPSE, oropharyngeal swallow efficiency; WD, with denture; WOD, without denture.

OPSE observed after dentures were worn can be considered attributable to changes in masticatory performance or sensation of oral cavity due to denture wearing.

In the present study, delays in OTTs were observed after denture wearing. Sensory inputs in the oral cavity including tastes play important roles in normal control of voluntary swallowing $[2,10,11]$. Local oropharyngeal anesthesia reduces senses or motor activities $[2,12,13]$, and dentures or implants reduce sensation in the oral cavity [14].

Improper denture wearing increases swallowing duration [1] and this change differs between complete dentures and partial dentures $[15,16]$. When dentures are removed, the tip of the tongue protrudes and thus the upward movements of the hyoid and the anterior movements of the larynx occur more easily then when dentures were worn [17], and as a result of this change, the opening of the upper esophageal sphincter increases and swallowing duration shortened [18]. Decreases in swal- lowing durations of edentulous patients were observed when dentures were removed compared to when dentures were worn [19]. Declines in sensation of oral cavity due to denture wearing, instability due to improper denture wearing or changes in tongue movements caused delays in OTTs. Although not considered in the present study, evaluation of the instability of dentures should be conducted to individually approach the effects of the instability of dentures on swallowing functions and the effects of declines in sensation of oral cavity on swallowing functions. Besides, since aging deteriorates swallowing functions [20], subgroup analysis by age is also necessary. In the present study, OPSE tending to decrease was observed when dentures were worn although the decreases were not statistically significant.

OPSE is a method to quantify the ability to transport foods from the oral cavity and the pharynx to the esophagus [21], and is closely correlated with OTT [22]. It may decrease when the amount of swallowed foods decreases, 
esophageal aspiration increases or passing times (OTT, PTT) increase [22]. Based on the foregoing, it is thought that denture wearing may reduce the swallowing volume or increase aspiration. However, as seen in the present study, decreases in OPSE due to delay in OTTs should be also considered besides denture wearing.

Airway protection is very important in swallowing. If the airway is not protected, aspiration may occur resulting in serious complications. In the present study, changes of aspiration were checked depending on denture wearing. When dentures were worn, four out of the entire 20 subjects showed aspiration and when dentures were removed, aspiration occurred in only two out of the four who showed aspiration when dentures were worn and not in the remaining two. Oropharyngeal local anesthesia causes oropharyngeal dysphagia and even aspiration [23]. Possibly sensory declines in the oral cavity due to denture wearing cause aspiration. However, in the present study, the association was not statistically significant since the number of patients in whom aspiration occurred was not large enough. However, the association between denture wearing and aspiration can be deduced from the fact that when dentures were removed aspiration did not newly occur in any patients and that aspiration did not occur in two out of four subjects in whom aspiration occurred when dentures were worn. Additional studies are necessary.

Limitations of the present study are: first, it is a single center study. Second, the order of states where denture were worn and the states where debenture were removed was not randomly set. Third, in the case of patients with unclear consciousness, studies were not accurately conducted because these patients did not cooperate well in the two point discrimination test and the evaluation of masticatory performance. Fourth, subgroups were not set based on the types of dentures, materials, sizes and installed positions and thus biases due to the foregoing could not be removed. Fifth, the results of the present study are limited to the dysphagia diet I used in the tests. Therefore, if these limitations are complemented and randomized studies are conducted with different types of dentures and different levels of viscosity of diets, more meaningful study results for the effects of dentures on swallowing may be produced.

In the present study, changes in functional dysphagia scales between when dentures were worn and when dentures were removed were analyzed among patients who were using dentures. Due to denture wearing, OTTs were delayed and OPSE showed a decreasing tendency although the decreases were not statistically significant. These changes were significantly associated with masticatory performance and sensation of the oral cavity.

\section{CONFLICT OF INTEREST}

No potential conflict of interest relevant to this article was reported.

\section{REFERENCES}

1. Monaco A, Cattaneo R, Masci C, Spadaro A, Marzo G. Effect of ill-fitting dentures on the swallowing duration in patients using polygraphy. Gerodontology 2012;29:e637-44.

2. Chee C, Arshad S, Singh S, Mistry S, Hamdy S. The influence of chemical gustatory stimuli and oral anaesthesia on healthy human pharyngeal swallowing. Chem Senses 2005;30:393-400.

3. Aviv JE, Martin JH, Sacco RL, Zagar D, Diamond B, Keen MS, et al. Supraglottic and pharyngeal sensory abnormalities in stroke patients with dysphagia. Ann Otol Rhinol Laryngol 1996;105:92-7.

4. Kidd D, Lawson J, Nesbitt R, MacMahon J. Aspiration in acute stroke: a clinical study with videofluoroscopy. Q J Med 1993;86:825-9.

5. Kazunori I, Kentaro M, Ken-ichi M, Tomohiro H, Takashi N. Reproducibility and accuracy in measuring masticatory performance using test gummy jelly. Prosthodont Res Pract 2005;4:9-15.

6. Guilleminault C, Li K, Chen NH, Poyares D. Two-point discrimination in patients with upper airway resistance syndrome, obstructive sleep apnea syndrome, and normal control subjects. Chest 2002;122;866-70.

7. Kuhlemeier KV, Yates P, Palmer JB. Intra- and interrater variation in the evaluation of videofluorographic swallowing studies. Dysphagia 1998;13:142-7.

8. Logemann JA. Manual for the videofluorographic study of swallowing. 2nd ed. Austin: Pro-Ed Inc.; 1993.

9. Hwang $\mathrm{CH}$, Choi KH, Ko YS, Leem CM. Pre-emptive swallowing stimulation in long-term intubated patients. Clin Rehabil 2007;21:41-6.

10. Miller AJ. Deglutition. Physiol Rev 1982;62:129-84. 
11. Cook IJ. Normal and disordered swallowing: new insights. Baillieres Clin Gastroenterol 1991;5:245-67.

12. Teismann IK, Steinstrater O, Warnecke T, Suntrup S, Ringelstein EB, Pantev C, et al. Tactile thermal oral stimulation increases the cortical representation of swallowing. BMC Neurosci 2009;10:71.

13. Tei K, Yamazaki Y, Kobayashi M, Izumiyama Y, Ono M, Totsuka Y. Effects of bilateral lingual and inferior alveolar nerve anesthesia effects on masticatory function and early swallowing. Oral Surg Oral Med Oral Pathol Oral Radiol Endod 2004;97:553-8.

14. Jang KS, Kim YS. Comparison of oral sensory function in complete denture and implant-supported prosthesis wearers. J Oral Rehabil 2001;28:220-5.

15. Tallgren A, Tryde G. Chewing and swallowing activity of masticatory muscles in patients with a complete upper and partial lower denture. J Oral Rehabil 1991;18:285-99.

16. Tallgren A, Tryde G. Swallowing activity of lip muscles in patients with complete upper and partial lower denture. J Oral Rehabil 1992;19:329-41.

17. Hattori F. The relationship between wearing complete dentures and swallowing function in elderly individuals: a videofluorographic study. Kokubyo Gakkai
Zasshi 2004;71:102-11.

18. Karacay S, Akin E, Sayin MO, Bulakbasi N. Real time balanced turbo field echo cine-MRI in the analysis of deglutition events and transit times. J Oral Rehabil 2006;33:646-53.

19. Gokce HS, Gokce SM, Akin E, Bulakbasi N, Akyol M. Effect of complete denture wearing on deglutition time: a cine-magnetic resonance imaging study. J Oral Rehabil 2012;39:198-209.

20. Yoshikawa M, Yoshida M, Nagasaki T, Tanimoto K, Tsuga $\mathrm{K}$, Akagawa Y. Influence of aging and denture use on liquid swallowing in healthy dentulous and edentulous older people. J Am Geriatr Soc 2006;54: 444-9.

21. Logemann JA, Kahrilas PJ, Kobara M, Vakil NB. The benefit of head rotation on pharyngoesophageal dysphagia. Arch Phys Med Rehabil 1989;70:767-71.

22. Rademaker AW, Pauloski BR, Logemann JA, Shanahan TK. Oropharyngeal swallow efficiency as a representative measure of swallowing function. J Speech Hear Res 1994;37:314-25.

23. Ertekin C, Kiylioglu N, Tarlaci S, Keskin A, Aydogdu I. Effect of mucosal anaesthesia on oropharyngeal swallowing. Neurogastroenterol Motil 2000;12:567-72. 